\title{
Cluster headache and neuropsychological functioning
}

Cephalalgia

32(II) 8I3-82

(C) International Headache Society 2012

Reprints and permissions:

sagepub.co.uk/journalsPermissions.nav DOI: 10.1 | 77/ 0333 |024|244993। cep.sagepub.com

@SAGE

\author{
Thomas Dresler ${ }^{1, *}$, Ralf Lürding ${ }^{2, *}$, \\ Yvonne Paelecke-Habermann ${ }^{3}$, Charly Gaul ${ }^{4}$, \\ Karsten Henkel ${ }^{5,6}$, Andrea Lindwurm-Späth ${ }^{2}$, Elke Leinisch ${ }^{2,7}$ \\ and Tim $P$ Jürgens ${ }^{8}$
}

\begin{abstract}
Background: Despite significant advances in unravelling the pathophysiology of cluster headache $(\mathrm{CH})$, little is known about neuropsychological functioning. Apart from neuroimaging studies indicating involvement of posterior hypothalamic and other areas frequently involved in nociception, some studies suggest involvement of prefrontal areas. Among others, these mediate executive functioning (EF).

Methods: Therefore, three neuropsychological tests (Trail Making Test (TMT), Go/Nogo Task and Stroop Task) were completed by four headache patient samples (chronic $\mathrm{CH}$, episodic $\mathrm{CH}$ in the active or inactive period, and migraine patients) and compared to healthy controls.

Results: Analyses revealed that patients especially with chronic and active episodic $\mathrm{CH}$ were particularly impaired in tests relying more on intact EF (i.e. TMT-B, Stroop interference) than on basal cognitive processes (i.e. TMT-A, Stroop naming). Within the $\mathrm{CH}$ groups performance decreased linearly with increasing severity.

Discussion: These findings are in line with a recently proposed involvement of prefrontal structures in $\mathrm{CH}$ pathophysiology as patients performed worse on neuropsychological tasks relying on these structures. Impaired EF could also result from medication and sleep disturbances due to active $\mathrm{CH}$. Because the decreased performance was also present outside the attacks it may hint at generally altered brain functions, but do not necessarily reflect clinically relevant behaviour.
\end{abstract}

\section{Keywords}

Cluster headache, neuropsychology, Trail Making Test, executive function, Stroop Task, migraine

Date received: 29 February 2012; revised: 27 April 2012; accepted: 6 May 2012

\section{Introduction}

Cluster headache $(\mathrm{CH})$ is characterized by recurring tormenting pain attacks (1) that impair a patient's functioning and quality of life to a great extent $(2,3)$. Recent studies on various aspects of impairment indicate that $\mathrm{CH}$ patients are severely impaired in economic as well as non-economic domains and seem to be more vulnerable to diverse psychiatric co-morbidities (4) with depression as a prevalent condition (5). Because $\mathrm{CH}$ pathology has also been associated with behavioural alterations (6), differences in more complex cognitive functions involving coordination, regulation and inhibition of behaviour are conceivable.

Neuroimaging studies in $\mathrm{CH}$ found morphological and functional changes in the posterior inferior hypothalamus (PIH) suggesting a key role in its pathophysiology (7), consistent with changes in

\footnotetext{
'Department of Psychiatry and Psychotherapy, University of Tübingen, Germany

${ }^{2}$ Department of Neurology, University of Regensburg, Germany

${ }^{3}$ Department of Psychology I, University of Würzburg, Germany

${ }^{4}$ Department of Neurology, University of Essen, Germany

${ }^{5}$ Kiel Headache Center, Kiel, Germany

${ }^{6}$ Department of Psychiatry, Psychotherapy and Psychosomatics, Medical School, RWTH Aachen University, Germany

${ }^{7}$ Department of Neurology, Helios Klinikum, Germany

${ }^{8}$ Department of Systems Neuroscience, University Medical Center

Hamburg-Eppendorf, Germany
}

*Thomas Dresler and Ralf Lürding contributed equally to this work.

\section{Corresponding author:}

Tim P Jürgens, Department of Systems Neuroscience, University Medical Center Hamburg-Eppendorf, Martinistrasse 52, D-20246 Hamburg,

Germany.

Email: t.juergens@uke.de 
neuroendocrinology reported in $\mathrm{CH}$ and good efficacy of hypothalamic deep brain stimulation in refractory $\mathrm{CH}$ (8). These data support a link between the PIH and the trigeminovascular system by nociceptive hypothalamo-trigeminal and hypothalamo-autonomic pathways. Little is known about connections to other regions such as the prefrontal cortex (PFC) via dopaminergic midbrain cells. Both PIH and PFC are part of a motivation circuit mainly involved in executive function (EF), impulse control and decision making (9). Interestingly, a study investigating episodic $\mathrm{CH}$ patients found a glucose hypometabolism in prefrontal structures both inside and outside the active period $\mathrm{CH}$ (10). Likewise, a study using visually-evoked eventrelated potentials (ERP) found increased latencies in $\mathrm{CH}$ patients that were interpreted as aberrancies in prefrontal brain areas (11).

So far, little is known about functional effects of prefrontal involvement on neuropsychological outcomes in $\mathrm{CH}$ such as EF (12). Impaired EF has been suggested for a variety of chronic pain conditions $(13,14)$, highlighting the association of pain syndromes with altered neuroplasticity and dysregulated neurochemistry in the PFC.

Whereas a general cognitive impairment in migraine and $\mathrm{CH}$ may be best seen during acute headache while wearing off interictally (15), data on specific functions such as memory, attention or EF are scarce. One study assessed verbal memory and $\mathrm{EF}$ in active episodic $\mathrm{CH}$ $(\mathrm{ECH})$ and reported only decreased auditory verbal memory as compared with tension headache patients (16), an impairment that could also be shown in migraine patients (17). As pain may interfere with cognitive performance (18), it is unclear whether pain itself (15) or the underlying headache pathology results in decreased test scores. It is not known either whether differences exist between the different subtypes of $\mathrm{CH}$ (such as episodic and chronic $\mathrm{CH}$ ).

In this study we hence aimed to determine whether there are specific differences between clinically important subgroups of $\mathrm{CH}$ in three neuropsychological tests designed for assessing monitoring, response inhibition and cognitive control (19-22), three important factors of EF (23). Successful performance in these tests requires the integrity of associated neuronal networks, especially prefrontal cortical areas (24-27).

\section{Methods}

\section{Participants}

Headache patients were recruited at the Departments of Neurology, University Hospitals of Regensburg and Halle, and at the Kiel Headache Centre as part of a larger study on different facets of cluster headache
$(4,28)$. Patients were diagnosed according to the ICHD-II criteria for migraine without and with aura (IHS 1.1 and 1.2) and for episodic and chronic cluster headache (IHS 3.1.1 and 3.1.2) by an experienced headache specialist (29). Ninety-seven headache patients were included, 27 patients with chronic cluster headache $(\mathrm{CCH}), 26$ with episodic cluster headache in the active period (ECHa), 22 with episodic cluster headache outside the active period (ECHi) and 24 patients with migraine (MIG). Additionally 31 healthy controls (HC) were recruited in the respective centres. Groups were comparable in age, patients were comparable in age of headache onset and duration of headache (for all tests: $p>0.12$ ). Patients in the ECH groups were allocated to one subgroup only and not examined twice (independent samples). Detailed data on the sample can be found elsewhere (4). All participants filled in the German version of the 'Alcohol Use Disorders Identification Test' (AUDIT) (30), a screening questionnaire to identify harmful drinking. All subjects gave written informed consent. The study was approved by the lead ethics committee of the University of Regensburg as well as the local ethic committees and was in compliance with the Declaration of Helsinki from 2008.

\section{Neuropsychological tests}

Neuropsychological testing included the Trail Making Test (TMT), the Go/Nogo Task and the Stroop Task. These tasks involve assessing the integrity of different neuropsychological functions, such as cognitive flexibility, monitoring, response inhibition and response execution, and cognitive control.

Trail Making Test (TMT). The TMT (31) consists of two conditions: the TMT-A requires participants to draw lines to connect, in order, circled numbers from 1 to 25 positioned randomly on a sheet of paper; the TMT-B requires participants to alternate between numbers and letters (e.g. $1-\mathrm{A}-2-\mathrm{B}-3-\ldots$ ). The processing time was measured in both TMT conditions. The TMT-B condition utilizes cognitive flexibility while switching between two tasks (numbers and letters), thus assessing monitoring (32). This is an important neuropsychological function that enables quick adoption of changing task requirements.

Go/Nogo Task. In the Go/Nogo Task, taken from a standardized attention test battery (33), participants have to respond to a Go stimulus while inhibiting the response to a Nogo stimulus. Median reaction time and standard deviation for Go trials and number of commission (responding in Nogo trials) and omission errors (non-responding in Go trials) were used as 
dependent variables. The Go/Nogo Task requires the suppression of an already prepared motor response, thus measuring response inhibition (34). This test involves both processes of response inhibition and response execution, thereby requiring higher-order motor and attention control.

Stroop Task (colour-word interference). Cognitive control was measured by the Stroop Task (35), a wellestablished neuropsychological tool (22). The German version (21) was used with the subtests reading, naming and interference. The interference condition necessitates selective turning towards a specific target feature (naming the colour) and control of automatic processes (reading the incongruent word). The time in colour reading, colour naming and interference, as well as two regression-corrected indices (nomination and selectivity) were used for statistical analyses. Nomination is an index for naming speed, corrected for reading speed (21). Selectivity is an index for concentrative resistance against dominating, automated response tendencies (reading), corrected for naming speed (21). Values above/below 100 indicate a shorter/longer RT than might be expected. This task requires the successful voluntary inhibition of involuntary automatic processes, an important facet of cognitive control.

\section{Data analysis}

Data were tested for normal distribution using the Kolmogorov-Smirnov test and analysed by means of analyses of variance (ANOVA) and post hoc $t$-tests (SPSS 18). If a normal distribution could not be ascertained, a non-parametric analysis (KruskalWallis test, Mann-Whitney $U$-test) was additionally conducted to check if they yielded identical results as the parametric tests. Non-parametric analyses will only be reported in case of divergence. If the assumption of sphericity was violated as shown in the Mauchly's test $(p<0.10)$, degrees of freedom were corrected according to Greenhouse-Geisser. When a condition was varied within a task, a univariate two-way (condition $\times$ group) ANOVA was performed. Interaction effects were further disentangled by univariate one-way ANOVAs and $t$-tests within the condition factor's levels. Bonferroni's correction was used to account for the accumulation of the alpha error; uncorrected $p$-values are given whenever necessary. Error data in the Go/Nogo task were analysed by non-parametric tests throughout. Alpha level was set to $5 \%$; all tests were twotailed. Because of missing data for some patients in the neuropsychological tests the number of subjects within a group can be smaller than written above (see Table 1).

Table I. Descriptive statistics and results of the univariate analyses for the neuropsychological variables.

\begin{tabular}{|c|c|c|c|c|c|c|}
\hline Variable & $\mathrm{CCH}$ & $\mathrm{ECHa}$ & $\mathrm{ECHi}$ & MIG & Control & $p$-value \\
\hline Trail-Making Test & $n=24$ & $n=26$ & $n=18$ & $n=23$ & $n=31$ & \\
\hline TMT-A & $30.67 \pm 9.17$ & $29.50 \pm 12.29$ & $26.06 \pm 5.76$ & $25.74 \pm 10.51$ & $24.10 \pm 8.76$ & $<0.001$ \\
\hline TMT-B & $89.96 \pm 36.60$ & $76.38 \pm 32.63$ & $61.11 \pm 15.33$ & $57.87 \pm 21.1 \mathrm{I}$ & $51.35 \pm 13.83$ & $<0.001$ \\
\hline Go/Nogo Task & $n=27$ & $n=26$ & $n=21$ & $n=22$ & $n=31$ & \\
\hline Commission errors & $2.15 \pm 3.76$ & $1.27 \pm 2.95$ & $1.19 \pm 3.93$ & $0.64 \pm 1.53$ & $0.61 \pm 1.02$ & $0.148^{a}$ \\
\hline Omission errors & $1.04 \pm 2.36$ & $0.50 \pm 1.42$ & $0.81 \pm 2.64$ & $0.55 \pm 2.56$ & $0.00 \pm 0.00$ & $0.006^{a}$ \\
\hline Go-RT & $572.24 \pm 90.34$ & $557.37 \pm 93.57$ & $529.24 \pm 54.47$ & $529.89 \pm 77.31$ & $499.82 \pm 74.05$ & 0.010 \\
\hline SD Go-RT & $94.27 \pm 37.60$ & $82.66 \pm 36.81$ & $75.78 \pm 21.79$ & $77.47 \pm 23.90$ & $68.37 \pm 25.45$ & 0.027 \\
\hline Stroop Task & $n=26$ & $n=25$ & $n=21$ & $n=23$ & $n=31$ & \\
\hline Stroop reading & $32.96 \pm 5.86$ & $30.92 \pm 4.81$ & $30.38 \pm 4.18$ & $29.09 \pm 4.65$ & $28.29 \pm 4.76$ & 0.008 \\
\hline Stroop naming & $48.85 \pm 7.15$ & $47.56 \pm 10.62$ & $45.29 \pm 9.27$ & $45.13 \pm 7.90$ & $4 I .13 \pm 5.91$ & 0.007 \\
\hline Stroop interference & $83.50 \pm 16.03$ & $78.84 \pm 20.81$ & $72.76 \pm 13.83$ & $74.39 \pm 12.96$ & $65.65 \pm 11.40$ & $<0.001$ \\
\hline Stroop nomination & $101.73 \pm 7.41$ & $100.80 \pm 6.78$ & $102.24 \pm 7.01$ & $100.04 \pm 5.72$ & $102.68 \pm 5.77$ & 0.610 \\
\hline Stroop selectivity & $100.35 \pm 6.73$ & $101.48 \pm 5.49$ & $102.19 \pm 5.90$ & $100.74 \pm 6.54$ & $101.68 \pm 4.13$ & 0.810 \\
\hline
\end{tabular}

Mean values and standard deviations are given.

${ }^{a}$ Kruskal-Wallis $\mathrm{H}$-test, in all other variables one-way ANOVAs were used.

$\mathrm{CCH}$ : chronic cluster headache; ECHa: episodic cluster headache in the active period; $\mathrm{ECHi:} \mathrm{episodic} \mathrm{cluster} \mathrm{headache} \mathrm{outside} \mathrm{the} \mathrm{active} \mathrm{period;}$ MIG: migraine; Control: healthy controls. 


\section{Results}

\section{Trail-Making Test (see Figure I)}

The $2 \times 5$ ANOVA with the factors condition (TMT-A, TMT-B) and group (CCH, ECHa, ECHi, M, HC) revealed a significant main effect for condition $\left(F_{1,117}=367.677, p<0.001\right)$ and group $\left(F_{4,117}=8.791\right.$, $p<0.001$ ), and a significant condition-by-group interaction $\left(F_{4,117}=8.236, p<0.001\right)$. The effect condition reflects the higher difficulty of the TMT-B with substantially increased response latencies apparent in all groups. The interaction was due to a differential effect of the groups within the two conditions. Post hoc oneway ANOVAs within the two conditions with the factor group revealed that only for the TMT-B the group factor was significant $\left(F_{4,117}=9.527, p<0.001\right)$. In the TMT-B, the $\mathrm{CCH}$ group differed significantly from the ECHi, MIG and $\mathrm{HC}$ group (all Bonferronicorrected $p<0.005$ ), but not from the ECHa group, which also differed from the HC group $(p=0.004)$. Descriptive statistics are given in Table 1.

\section{Go/Nogo Task (see Figure 2)}

The Kruskal-Wallis $H$-test did not reveal a significant effect for the commission errors $\left(\chi_{4}{ }^{2}=6.788\right.$, $p=0.148$ ), that would have indicated an impulsive or disinhibited reaction, but only for omission errors $\left(\chi_{4}^{2}=14.453, p=0.006\right)$. That was due to more omission errors in all three $\mathrm{CH}$ groups as compared to the controls (Mann-Whitney $U$-tests: $\mathrm{p}<0.05$ ). For the reaction time data a significant effect of group was found in the ANOVA for the median reaction time (i.e. decision speed, $F_{4,122}=3.499, p=0.010$ ), which was due to a significantly increased reaction time for the $\mathrm{CCH}$ compared to the $\mathrm{HC}$ group (Bonferronicorrected $p=0.008)$. At an uncorrected level also ECHa differed from the HC group $(p<0.01)$. For the standard deviation of the individual reaction times a significant group effect emerged $\left(F_{4,122}=2.835\right.$, $p=0.027$ ), which was also due to a significant increase in the $\mathrm{CCH}$ compared to the HC group $(p<0.05)$. At an uncorrected level $\mathrm{CCH}$ also differed from the ECHi group $(p<0.05)$. Descriptive statistics are given in Table 1.

\section{Stroop Task (see Figure 3)}

For the Stroop interference task the $3 \times 5$ ANOVA with the factors condition (reading, naming, interference) and group revealed a significant main effect for condition $\left(F_{1.3,157.6}=1143.148, p<0.001\right)$ and group

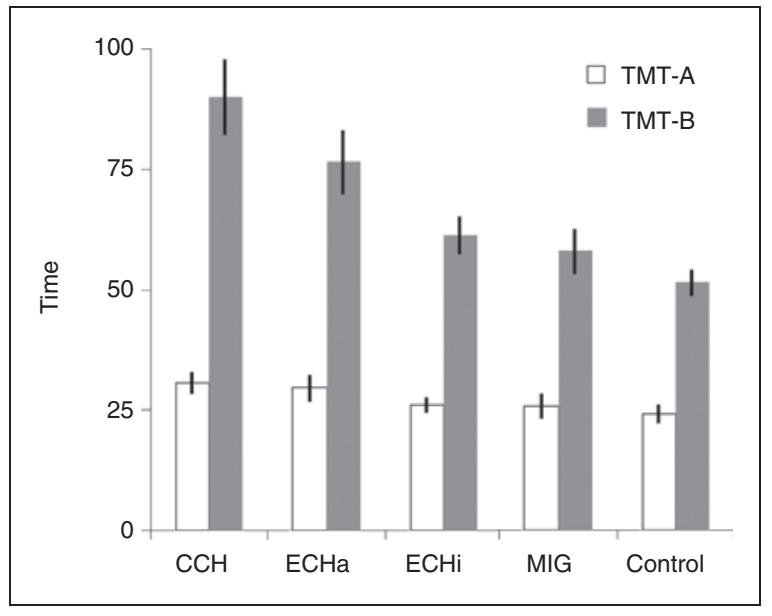

Figure I. Mean processing time in the Trail-Making Test (TMT-A and TMT-B). Error bars indicate standard errors of the mean. $\mathrm{CCH}$ : chronic cluster headache; $\mathrm{ECHa}$ : episodic cluster headache in the active period; ECHi: episodic cluster headache outside the active period; MIG: migraine; Control: healthy controls.

$\left(F_{4,121}=5.651, p<0.001\right)$, and a significant interaction condition-by-group $\left(F_{5.2,157.6}=3.582, p=0.004\right)$. The interaction effect was due to a larger group effect in the interference than in the other two conditions as two-way ANOVAs with two conditions were only significant when the interference condition was included $\left(F_{4,121}>3.907, p<0.01\right)$, but not for the remaining two conditions (reading, naming; $F_{4,121}=1.404, p=0.237$ ). Descriptive statistics are given in Table 1.

One-way ANOVAs within each condition revealed a significant group effect for all three conditions: reading $\left(F_{4,121}=3.656, \quad p<0.01\right)$, naming $\left(F_{4,121}=3.696\right.$, $p<0.01)$, and interference $\left(F_{4,121}=5.428, p<0.001\right)$. In all conditions the Bonferroni-corrected post hoc tests revealed that the $\mathrm{CCH}$ group showed significantly increased response latencies as compared to the $\mathrm{HC}$ group; in naming and interference the $\mathrm{ECHa}$ group also showed significantly increased response latencies. At an uncorrected level MIG patients had an increased interference $(p=0.04)$ and naming time $(p=0.08)$ as compared to controls

The two additional indices derived from the data (21), i.e. nomination (corrected nomination speed) and selectivity score (corrected interference score) did not reveal any differences between the groups.

To investigate neuropsychological functioning within the three $\mathrm{CH}$ groups, we set up linear polynomial contrasts (i.e. -101 ) for each of the indices assuming linear effects from ECHi over ECHa to $\mathrm{CCH}$. These were significant for the TMT-B and the Stroop interference (all $t>2.115 ; p<0.05$ ). These tests indicate increased EF impairment with more severe $\mathrm{CH}$ (see Figures 1-3). 


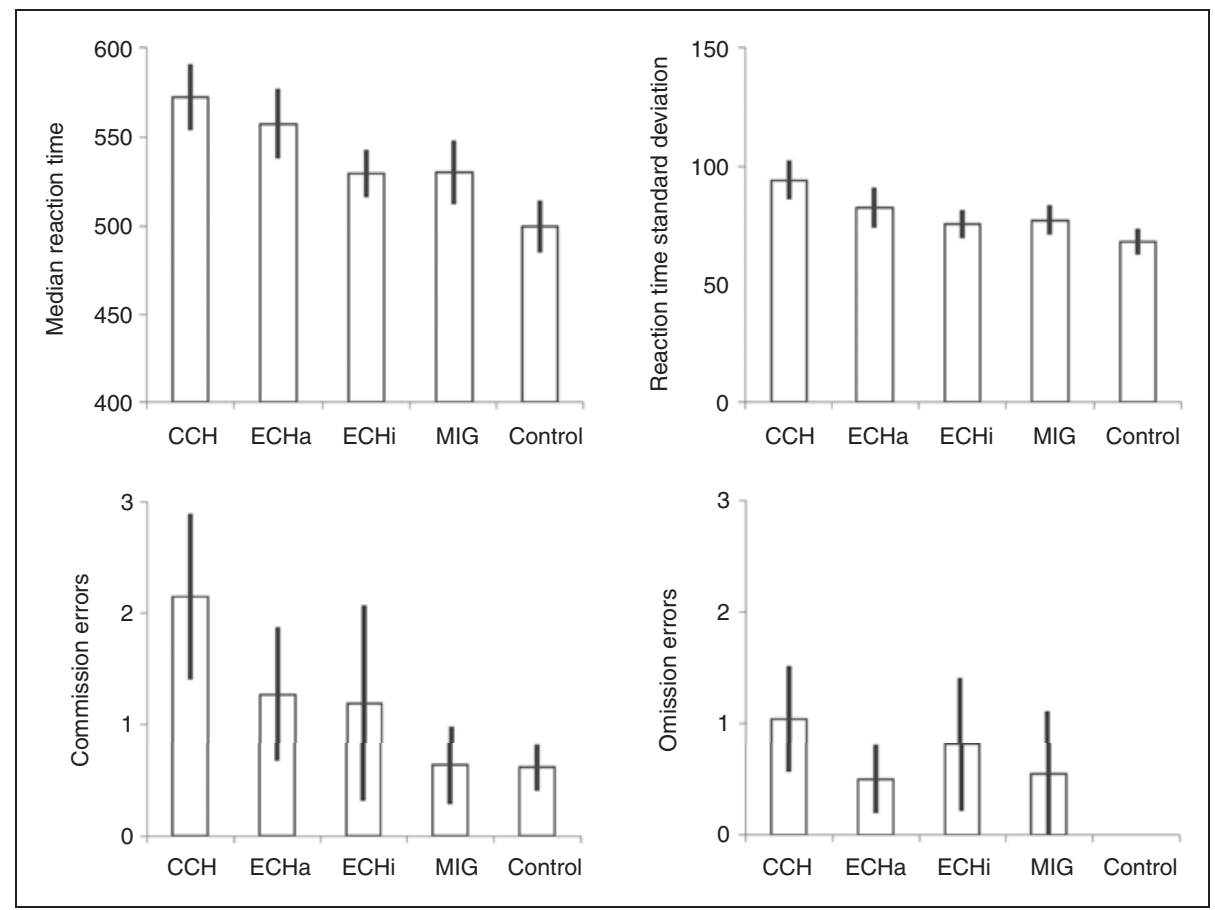

Figure 2. Median reaction time, standard deviation (upper panels), commission and omission errors (lower panels) in the Go/Nogo Task. Error bars indicate standard errors of the mean.

$\mathrm{CCH}$ : chronic cluster headache; ECHa: episodic cluster headache in the active period; ECHi: episodic cluster headache outside the active period; MIG: migraine; Control: healthy controls.

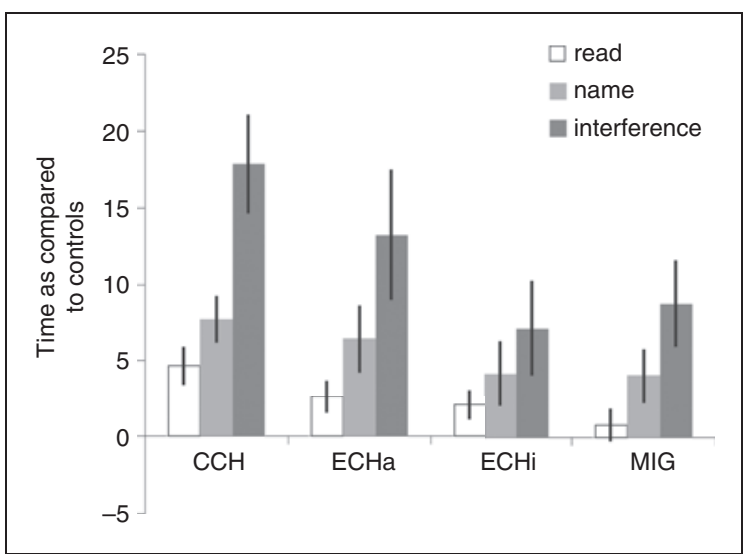

Figure 3. Mean time needed for the subtests colour reading, colour naming and interference in the Stroop Task (colour-word interference) as compared to healthy controls. Error bars indicate standard errors of the mean.

$\mathrm{CCH}$ : chronic cluster headache; $\mathrm{ECH}$ : episodic cluster headache in the active period; $\mathrm{ECHi}$ : episodic cluster headache outside the active period; MIG: migraine.

\section{Investigation of potential confounds}

To elucidate the influence of potential confounds on the neuropsychological variables, influence of medication, attack frequency, self-reported lifetime depression, circadian timing of attacks and alcohol consumption were investigated.

Various prophylactics (i.e. verapamil, lithium, gabapentin, topiramate, valproic acid and beta-blockers) were taken by $22 \mathrm{CCH}$ patients, $17 \mathrm{ECHa}$ patients, 5 ECHi patients and 6 migraine patients (see Table 2 for further details).

Within the $\mathrm{CH}$ groups, taking prophylactic medication hardly influenced neuropsychological performance. Although patients with ECHi taking prophylactic medication $(n=5)$ performed worse in the TMT-A and TMT-B (Mann-Whitney $U$-test: $p=0.019 / 0.063$ ), results did not differ significantly in the other groups.

In the combined group of $\mathrm{CH}$ patients there was no significant association of the neuropsychological variables with attack frequency, days on which acute medication is taken per month and amount of daily acute medication (Spearman's $\rho$, all $p>0.05$, uncorrected).

In the combined group of $\mathrm{CH}$ patients, patients reporting lifetime depressive symptoms showed increased GoNogo reaction time and standard deviation $(t>2.74, p<0.05)$ when compared with patients who did not report such symptoms. As distribution of self-reported lifetime depressive symptoms was just equally distributed $\left(\chi^{2}=4.50, p=0.11\right)$, additional exploratory analysis revealed that within the groups no consistent patterns could be confirmed. 
Table 2. Preventative drugs used by patients

\begin{tabular}{llcll}
\hline Variable & $\begin{array}{l}\mathrm{CCH} \\
n=27\end{array}$ & $\begin{array}{l}\mathrm{ECHa} \\
n=26\end{array}$ & $\begin{array}{l}\mathrm{ECHi} \\
n=22\end{array}$ & $\begin{array}{l}\text { MIG } \\
n=24\end{array}$ \\
\hline Verapamil & $\mathrm{I}(6 \mathrm{67 \% )}$ & $\mathrm{I} 5(58 \%)$ & $4(\mathrm{I} \%)$ & 0 \\
Lithium & $4(15 \%)$ & $5(19 \%)$ & 0 & $3(\mathrm{I} \%)$ \\
Topiramate & $2(7 \%)$ & $\mathrm{I}(4 \%)$ & $\mathrm{I}(5 \%)$ & 0 \\
Gabapentin & 0 & $\mathrm{I}(4 \%)$ & 0 & 0 \\
Beta-blocker & $\mathrm{I}(4 \%)$ & 0 & 0 & $3(13 \%)$
\end{tabular}

$\mathrm{CCH}$ : chronic cluster headache; $\mathrm{ECHa}$ : episodic cluster headache in the active period; $\mathrm{ECHi}$ : episodic cluster headache outside the active period; MIG: migraine.

When patients with daily attacks were contrasted with patients with nocturnal or both daily and nocturnal attacks to exclude sleep deprivation as a confounder, no significant difference was found.

As excessive alcohol consumption is associated with deficits in executive functions (e.g. $(36,37)$ ), differences in alcohol use might have influenced the results. We reanalysed the data using the presence of hazardous drinking as detected with the AUDIT (defined by a threshold sum score of 5). Results for the primary EF variables were not changed.

Taken together, no consistent patterns were found that indicate a substantial influence of putative confounds on the neuropsychological variables.

\section{Discussion}

In the present study neuropsychological functioning in $\mathrm{CH}$ was investigated and the results showed that chronic $\mathrm{CH}$ and episodic $\mathrm{CH}$ patients in the active period were particularly impaired in tests relying more on intact executive function than on basal cognitive processes.

\section{Neuropsychological functioning in $\mathrm{CH}$}

In all neuropsychological measures (Trail-Making Test, Go/Nogo Task and Stroop Task) the analyses yielded significant effects indicating meaningful group differences between patients with $\mathrm{CH}$, migraine and healthy controls. Post hoc tests revealed that the differences were mainly driven by the $\mathrm{CCH}$ group performing worse as compared to the controls. The results also revealed a pattern of worse performance with increasing severity of $\mathrm{CH}$, as the performance scores were arranged in the order $\mathrm{CCH}>\mathrm{ECHa}>\mathrm{ECHi}$. Such a pattern could be seen in almost every variable, even when the analysis failed to reach significance. Hence, across different presentations of $\mathrm{CH}, \mathrm{CCH}$ in particular was associated with decreased performance. Interestingly, and in line with our hypotheses, the effects were more pronounced for subtests that mainly rely on EF, respectively monitoring and cognitive control, demanding increased cognitive capacity such as the TMT-B or the Stroop interference condition, whereas more basic cognitive tasks (TMT-A, Stroop reading, Stroop naming) were performed more equally (see Figures 1 and 3). For the nomination and selectivity scores in the Stroop Task, i.e. scores corrected for reading and naming speed, respectively, no significant group differences emerged. This may indicate that even basic processes necessary for complex EF could be affected. However, as these scores are scarcely reported and only established in German samples, the generalizability of these results remains preliminary and warrant further investigation. The uncorrected processing time in the interference condition - normally used as a measure for EF in other studies - indicates decreased performance in cognitive control in the $\mathrm{CCH}$ patients. There is a need to discuss why the EF index in the $\mathrm{Go} /$ Nogo task (i.e. commission errors as usual indicator of response inhibition) did not differ between the groups, whereas an increased response time in the Go condition and an increased number of omission errors were found in the $\mathrm{CCH}$ patients. This may point towards an increased response inhibition. It is feasible that - while monitoring (TMT-B) and cognitive control (Stroop) is impaired - patients tend to respond more carefully in tasks measuring impulsivity/inhibition to avoid making mistakes. That is, they prefer responding more slowly and omitting a response than committing a wrong response. This corresponds with the finding that $\mathrm{CH}$ patients are aware of their condition and the resulting impairment (4).

Data on neuropsychological characteristics of patients with $\mathrm{CH}$ are scarce. Jorge et al. (16) found decreased verbal memory scores in ECHa patients, which the authors assumed to be the result of a putatively impaired left medial temporal network function. However, they did not report differences in EF, which yet might be due to the included tests (Rey Complex Figure Test, Tower of Toronto, Oral Word Association Test) and to a control group of tension headache patients - which might underestimate a decrease in performance. Besides, $\mathrm{CCH}$ patients - the group performing worst in the present study - were not studied. Imaging studies rather hint towards a prefrontal involvement in the pathophysiology of $\mathrm{CH}(1,10,11)$. Likewise, in our study altered interictal responses in prefrontal brain function resulting in impaired test results could be assumed a priori. In line with a previous imaging study (10) that found hypometabolism in $\mathrm{CH}$ inside, but also outside the bout, $\mathrm{CH}$ patients performed poorly in tasks strongly relying on these prefrontal structures. Given this, the neuropsychological profile found in this study (i.e. $\mathrm{CH}$ patients display a 
decreased neuropsychological performance) fits well with the underlying reported neurometabolical changes. This is a pathophysiologically relevant issue that has not been addressed before. The decreased performance does not imply that it interferes with normal daily activities, as these tests assess more subtle neuropsychological dysfunction. A detailed analysis of the relation between subtle cognitive effects and selective influences in quality of life would overemphasize the results of this study. For tests assessing more severe cognitive impairment with ceiling effects (such as the Mini Mental State Examination) decreased performance in $\mathrm{CH}$ patients seems to be only apparent during headache episodes, but not in between (15). Due to the excruciating nature of the $\mathrm{CH}$ attacks, neuropsychological assessment during attacks is not feasible. Thus, neuropsychological tests measuring EF should be used interictally to assess a possible prefrontal impairment. As one study found, executive dysfunction may predict the formation of psychiatric symptoms (38) which are frequent in patients with $\mathrm{CH}(4)$ and thus in the future a well-chosen test battery may represent a tool to detect patients at risk at an early stage. However, up to now, these considerations are speculative and to draw valid conclusions more longitudinal data are needed. Also, the reported study (38) only investigated psychiatric inpatients, thereby limiting the generalizability of the results.

\section{Neuropsychological functioning in migraine patients}

Migraine patients did not substantially differ from the healthy controls in our examination; only a worse performance in Stroop interference emerged at an uncorrected alpha level. So far, neuropsychological findings in migraine patients are not conclusive. Whereas some studies found worse performance (especially in memory), others did not (for a review see (39)). The largest effects were found for the $\mathrm{CCH}$ and $\mathrm{ECHa}$ groups, further supporting the headache severity in these groups. These results are hence in line with a recent review article that found only weak or inconclusive evidence for impaired neuropsychological functioning in migraine patients (40).

\section{Neuropsychological function in painful conditions}

Pain experience, itself resulting in decreased neuropsychological performance, might offer an explanation at first sight, as such effects have been found in pain challenges in healthy controls (18). Yet these studies focus more on the impact of acute pain rather than chronic pain present for years. It is therefore unlikely that results can be explained by experience of pain itself rather than a specific effect of $\mathrm{CH}$.

\section{Limitations}

Although we aimed to control for the potential confounders 'medication' and 'sleep disturbance', we actually cannot rule out an influence of these factors. The percentage of patients taking prophylactic medication in the $\mathrm{CCH}$ and $\mathrm{ECHa}$ groups was higher than in the ECHi group, which might have influenced the results. We also did not directly assess sleep disturbances by use of a sleep diary, somnography or direct interrogation but relied on information about the timing of attacks. More homogeneous groups would have been needed to exclude or control for cognitive effects of each preventative drug as relevant confounder. Studies on cognitive effects of verapamil (41) and topiramate (42) are sparse and do not allow any comparison with data presented in this study. Although the factors 'medication' and 'sleep disturbance' did not show any relevant effect on EF, more appropriate variables are necessary to fully examine these complex relationships. In future studies longitudinal designs should additionally be considered to better elucidate such effects.

One limitation of the present study is a sample size of about 20 to 25 subjects per group, which restricts the validity of extensive correlation analyses. Larger studies will help to find possible associations of the neuropsychological performance with other variables more reliably. However, this is the first study with wellcharacterized headache patients including the clinically relevant subgroups of $\mathrm{CH}$ totalling almost 100 patients. The additional use of neuroimaging methods would have helped to further corroborate the interaction between neuropsychological functioning and underlying pathophysiology.

As only self-reported symptoms of lifetime depression were recorded, confounding effects of acute depressive symptoms were not assessed. In addition, the number of depressive episodes should be recorded in future studies, as neuropsychological performance decreases with increasing number of episodes (43).

We cannot rule out that the selection of neuropsychological tests may have influenced the results, as other tests might have been more sensitive (e.g. memory tests as in (16)). However, derived from recent imaging data in $\mathrm{CH}$, the applied tests seem to map the neural alterations best. 


\section{Conclusion}

Our data suggest prefrontal alterations in patients with $\mathrm{CH}$ that are reflected in the performance in neuropsychological tasks measuring EF and are in line with recent neuroimaging studies. Because the decreased performance was present outside the attacks it may hint at generally altered brain functions. Although these changes do not necessarily reflect clinically relevant behaviour, they contribute to a better understanding of $\mathrm{CH}$ pathophysiology.

\section{Funding}

$\mathrm{KH}$ has received honoraria from Gruenenthal. CG has received research grants and honoraria from MSD, Berlin Chemie, Desitin, St. Jude, Medtronic, Allergan and Böhringer Ingelheim. TPJ has received grants and honoraria from MSD.

\section{Acknowledgements}

This study was conducted by members of the DMKG (German Migraine and Headache Society) network of young researchers.

\section{References}

1. May A. Cluster headache: pathogenesis, diagnosis, and management. Lancet 2005; 366: 843-855.

2. D'Amico D, Rigamonti A, Solari A, et al. Health-related quality of life in patients with cluster headache during active periods. Cephalalgia 2002; 22: 818-821.

3. Jensen RM, Lyngberg A and Jensen RH. Burden of cluster headache. Cephalalgia 2007; 27: 535-541.

4. Jürgens TP, Gaul C, Lindwurm A, et al. Impairment in episodic and chronic cluster headache. Cephalalgia 2011; 31: 671-682.

5. Seifert CL, Valet M, Pfaffenrath V, et al. Neurometabolic correlates of depression and disability in episodic cluster headache. $J$ Neurol 2011; 258: 123-131.

6. Torelli P and Manzoni GC. Behavior during cluster headache. Curr Pain Headache Rep 2005; 9: 113-119.

7. May A, Leone M, Boecker $\mathrm{H}$, et al. Hypothalamic deep brain stimulation in positron emission tomography. J Neurosci 2006; 26: 3589-3593.

8. Leone M, Proietti Cecchini A, Franzini A, et al. Lessons from 8 years' experience of hypothalamic stimulation in cluster headache. Cephalalgia 2008; 28: 787-797; discussion 98 .

9. Munte TF, Heldmann M, Hinrichs H, et al. Contribution of subcortical structures to cognition assessed with invasive electrophysiology in humans. Front Neurosci 2008; 2: 72-78.

10. Sprenger $\mathrm{T}$, Ruether $\mathrm{KV}$, Boecker $\mathrm{H}$, et al. Altered metabolism in frontal brain circuits in cluster headache. Cephalalgia 2007; 27: 1033-1042.

11. Evers S, Bauer B, Suhr B, et al. Cognitive processing is involved in cluster headache but not in chronic paroxysmal hemicrania. Neurology 1999; 53: 357-363.
12. Dubois B, Andrade K and Levy R. Executive dysfunction and neuropsychological testing. Handb Clin Neurol 2008; 89: $35-52$.

13. Hart RP, Martelli MF and Zasler ND. Chronic pain and neuropsychological functioning. Neuropsychol Rev 2000; 10: 131-149.

14. Moriarty O, McGuire BE and Finn DP. The effect of pain on cognitive function: a review of clinical and preclinical research. Prog Neurobiol 2011; 93: 385-404.

15. Meyer JS, Thornby J, Crawford K, et al. Reversible cognitive decline accompanies migraine and cluster headaches. Headache 2000; 40: 638-646.

16. Jorge RE, Leston JE, Arndt S, et al. Cluster headaches: association with anxiety disorders and memory deficits. Neurology 1999; 53: 543-547.

17. Calandre EP, Bembibre J, Arnedo ML, et al. Cognitive disturbances and regional cerebral blood flow abnormalities in migraine patients: their relationship with the clinical manifestations of the illness. Cephalalgia 2002; 22: 291-302.

18. Nicholson K, Martelli MF and Zasler ND. Does pain confound interpretation of neuropsychological test results? Neurorehabilitation 2001; 16: 225-230.

19. Chaytor N, Schmitter-Edgecombe M and Burr R. Improving the ecological validity of executive functioning assessment. Arch Clin Neuropsychol 2006; 21: 217-227.

20. Suchy Y, Leahy B, Sweet JJ, et al. Behavioral Dyscontrol Scale deficits among traumatic brain injury patients, part II: Comparison to other measures of executive functioning. Clin Neuropsychol 2003; 17: 492-506.

21. Bäumler G. Farb-Wort-Interferenztest (FWIT) nach J.R. Stroop. Göttingen: Hogrefe, 1985.

22. Cohen JD, Dunbar K and McClelland JL. On the control of automatic processes: a parallel distributed processing account of the Stroop effect. Psychol Rev 1990; 97: 332-361.

23. Miyake A, Friedman NP, Emerson MJ, et al. The unity and diversity of executive functions and their contributions to complex "Frontal Lobe" tasks: a latent variable analysis. Cogn Psychol 2000; 41: 49-100.

24. Laird AR, McMillan KM, Lancaster JL, et al. A comparison of label-based review and ALE meta-analysis in the Stroop task. Hum Brain Mapp 2005; 25: 6-21.

25. Moll J, de Oliveira-Souza R, Moll FT, et al. The cerebral correlates of set-shifting: an fMRI study of the trail making test. Arq Neuropsiquiatr 2002; 60: 900-905.

26. Watanabe J, Sugiura M, Sato K, et al. The human prefrontal and parietal association cortices are involved in NO-GO performances: an event-related fMRI study. Neuroimage 2002; 17: 1207-1216.

27. Zakzanis KK, Mraz R and Graham SJ. An fMRI study of the Trail Making Test. Neuropsychologia 2005; 43: 1878-1886.

28. Lürding R, Henkel K, Gaul C, et al. Aggressiveness in different presentations of cluster headache - results from a controlled multicentric study. Cephalalgia 2012; accepted for publication.

29. Headache Classification Subcommittee of the International Headache Society. The International 
Classification of Headache Disorders: 2nd edition. Cephalalgia 2004; 24(Suppl 1): 9160.

30. Babor TF, Higgins-Biddle JC, Saunders JB, et al. The Alcohol Use Disorders Identification Test. Guidelines for use in primary care, 2nd edn. Geneva: World Health Organization, 2001.

31. Reitan RM. Validity of the Trail Making Test as an indicator of organic brain damage. Percept Mot Skills 1958; 8: $271-276$

32. Müller SV and Münte TF. Störungen von Exekutivfunktionen. In: Sturm W, Herrmann $\mathbf{M}$ and Münte TF (eds) Lehrbuch der Klinischen Neuropsychologie. Heidelberg: Spektrum, 2009.

33. Zimmermann $\mathrm{P}$ and Fimm B. Testbatterie zur Aufmerksamkeitsprüfung (TAP) Version 1.02c. Herzogenrath: Psychologische Testsysteme, 1993.

34. Simmonds DJ, Pekar JJ and Mostofsky SH. Metaanalysis of Go/No-go tasks, demonstrating that fMRI activation associated with response inhibition is taskdependent. Neuropsychologia 2008; 46: 224-232.

35 . Stroop JR. Studies of interference in serial verbal reactions. J Exp Psychol 1935; 28: 643-662.

36. Manning V, Wanigaratne S, Best D, et al. Changes in neuropsychological functioning during alcohol detoxification. Eur Addict Res 2008; 14: 226-233.
37. Dresler T, Schecklmann M, Ernst LH, et al. Recovery of cortical functioning in abstinent alcohol-dependent patients: Prefrontal brain oxygenation during verbal fluency at different phases during withdrawal. World $J$ Biol Psychiatry 2012; 13: 135-145.

38. Serper M, Beech DR, Harvey PD, et al. Neuropsychological and symptom predictors of aggression on the psychiatric inpatient service. J Clin Exp Neuropsychol 2008; 30: 700-709.

39. O'Bryant SE, Marcus DA, Rains JC, et al. The neuropsychology of recurrent headache. Headache 2006; 46: 1364-1376.

40. Suhr JA and Seng EK. Neuropsychological functioning in migraine: clinical and research implications Cephalalgia 2012; 32: 39-54.

41. Muldoon MF, Waldstein SR, Ryan CM, et al. Effects of six anti-hypertensive medications on cognitive performance. J Hypertens 2002; 20: 1643-1652.

42. Pandina GJ, Ness S, Polverejan E, et al. Cognitive effects of topiramate in migraine patients aged 12 through 17 years. Pediatr Neurol 2010; 42: 187-195.

43. Paelecke-Habermann Y, Pohl J and Leplow B. Attention and executive functions in remitted major depression patients. J Affect Disord 2005; 89: 125-135. 